\title{
A Cascade-Classifier Approach for Fall Detection
}

\author{
I Putu Edy Suardiyana \\ Putra \\ Cogent Labs \\ Coventry University \\ Coventry, United Kingdom \\ putrai@uni.coven- \\ try.ac.uk
}

\author{
James Brusey \\ Cogent Labs \\ Coventry University \\ Coventry, United Kingdom \\ j.brusey@coventry.ac.uk
}

\author{
Elena Gaura \\ Cogent Labs \\ Coventry University \\ Coventry, United Kingdom \\ e.gaura@coventry.ac.uk
}

\begin{abstract}
The current machine learning algorithms in fall detection, especially those that use a sliding window, have a high computational cost because they need to compute the features from almost all samples. This computation causes energy drain and means that the associated wearable devices require frequent recharging, making them less usable. This study proposes a cascade approach that reduces the computational cost of the fall detection classifier. To examine this approach, accelerometer data from 48 subjects performing a combination of falls and ordinary behaviour is used to train 3 types of classifier (J48 Decision Tree, Logistic Regression, and Multilayer Perceptron). The results show that the cascade approach significantly reduces the computational cost both for learning the classifier and executing it once learnt. Furthermore, the Multilayer Perceptron achieves the highest performance with precision of $93.5 \%$, recall of $94.2 \%$, and f-measure of $93.5 \%$.
\end{abstract}

\section{Categories and Subject Descriptors}

J.3 [Computer Applications]: Life and Medical Scienceshealth, medical information system

\section{General Terms}

Algorithms, Design, Experimentation, Performance

\section{Keywords}

Fall detection, Machine Learning, Cascade classifier, Computational cost

\section{INTRODUCTION}

Falls are one of the major leading causes of injury-related death and they are the main cause of disability and death for people aged over 65 in the UK [2]. Complications such as hypothermia or pneumonia can become a long term negative effect of falls [8]. Although fall detection cannot prevent falls, it may alleviate or reduce complications by going some way to ensure that fall victims receive help quickly.
Most of the current fall detection techniques use threshold based algorithms because of the efficiency of this technique. However, this produces a high number of false alarms because some activities (such as sitting down quickly, walking down the stairs, or lying on the bed) produce an acceleration as high as fall events [20].

Machine learning based algorithms have become popular in recent time because they are able to construct a classifier that can detect falls effectively. Research has shown that machine learning approaches are able to distinguish fall and non-fall activities with a high accuracy [7, 6, 18]. However, the disadvantage of machine learning based algorithms is that they need to extract the features from a sliding window that can cause a high computational cost issue. This issue makes them difficult to implement in wearable sensors that have limited resources.

To overcome the computational cost issue, we propose a cascade-classifier approach that has lower computational cost and better accuracy than a sliding window based machine learning algorithm. We tested our approach on a large dataset that contains various types of Activities of Daily Living (ADLs) and simulated falls from 48 healthy subjects between 18-51 years old [17]. J48 Decision Tree, Logistic Regression and Multilayer Perceptron were chosen to test our approach.

The rest of the paper is organised as follows. Section II presents related work in fall detection. The dataset that used in this research is described in Section III. Our approach is explained in Section IV. Section V discusses the results and Section VI gives conclusions as well as prospective future work.

\section{RELATED WORK}

Igual et al. [11] identifies two categories of fall detectors: context-aware systems and wearable devices. Context-aware systems use cameras, infrared, or floor sensors while the wearable devices are worn on the subject (such as a pendant, watch or belt). According to Gjoreski et al. [9], the context-aware systems face several challenges such as the sensors placement, flexibility, user privacy, and the price of the sensors. Therefore, the wearable devices may be a better alternative as they are inexpensive and small. Accelerometer and gyroscope sensors are the most common sensors in wearable fall detectors. Within such systems, there are generally two types of algorithm used: threshold-based and machine 
learning-based [11]. Threshold-based algorithms use a predefined value as a threshold to detect a fall $[4,14,21]$. Machine learning-based approaches use a supervised machine learning tool, such as a Support Vector Machine (SVM), Decision Tree, or Artificial Neural Network, to learn a classifier that can recognise a fall, based on either the raw sensor measurement or some feature that has been computed from the raw measurement $[6,7,15,18]$.

Although the threshold-based algorithms have a low computational cost, implementing them in real-world cases is a difficult task because the thresholds are usually configured based on simulated fall signals [3]. This makes them inappropriate for real-world falls. Therefore, machine learning based algorithms are a better alternative as they can be trained using simulated fall signals to get a general pattern of falls. However, these approaches incur a high computational cost because of feature calculation.

Kau and Chen [13] proposed a cascade-classifier approach with Support Vector Machine (SVM) classifier as a fall detector on the smartphone. Their study shows that their approach increases the efficiency of the machine learning algorithm for fall detection by reducing the number of feature calculations. Their approach used an accelerometer and a gyroscope that are embedded in a smartphone platform. Based on Abbate et al.[1], the problem with using a smartphone as a fall detector is users needs to carry their smartphone in a fixed position. In most cases, they prefer to have a small and non-invasive sensor so they can put their smartphone wherever they want. Therefore, this study focused on developing an approach that can learn patterns of falls from small and non-invasive wearable sensors.

\section{DATASET}

A large dataset from Ojetola et al. [17] was used in this study. The data were gathered from 48 (9 females and 39 males) healthy subjects where each of them was required to simulate 14 falls (forward-fall, backward-fall, lateral fall) and several ADLs for 23 minutes on average. A more detailed information regarding the protocol is described on Ojetola et al. [17].

Table 1 shows the profile of the subjects. The data were gathered from sensors that were strapped to the chest, wrist, and thigh of each subject. Some subjects also have data that were gathered from a sensor that was strapped to waist. In this study, we used only the chest sensor data because the chest is the best place to place a sensor for posture recognition [9].

Shimmer sensors with a sampling rate of $100 \mathrm{~Hz}$ were used as data collectors. The sensor consists of 3D accelerometer, 3D gyroscope, a Bluetooth device, and MSP430F1611 microcontroller device. The data were transferred to a Personal Computer (PC) using a Bluetooth device and they were annotated with a LabView program. Figure 1 shows the falls and some ADLs of this dataset.

\section{THE CASCADE-CLASSIFIER APPROACH 4.1 The Micro-annotation Approach}

In the window-based approach, the output of the classifier is often misalign with the data segment that is annotated
Table 1: Subject body profile summary

\begin{tabular}{ccccc}
\hline Profile & Minimum & Maximum & Average & Standard Deviation \\
\hline \hline Age (years) & 18 & 51 & 23.5 & 5.4 \\
Height $(\mathrm{cm})$ & 157 & 192 & 172.5 & 7.7 \\
Weight $(\mathrm{kg})$ & 45 & 108 & 69.4 & 12.7 \\
\hline
\end{tabular}

as a fall. It produces an ambiguity especially in the training and testing phases of the machine learning algorithm. Ojetola [16] proposed a micro-annotation approach to solve this problem. This approach re-annotates only one sample as a fall in each fall event segment. In our experiment, we adopted this approach to train and test our cascade-classifier approach. The illustrations of both the ambiguity of window based approach and the micro-annotation approach are shown in Figure 2.

\subsection{Features}

In calculating the features, three stages were used: preimpact, impact and post-impact. These three stages are useful to capture the fall history of the subject [16]. The lengths of pre-impact, impact, and post-impact are 1 second, 6 seconds, and 9 seconds. The length of the windows were chosen based on [16]. The following subsections explain the features that were used in our approach for their respective stages.

\subsubsection{Active State (Act_state)}

This feature was extracted based on the vector magnitude of three-axis acceleration outputs. Based on Abbate et al.[1], people in an inactive state (e.g sitting or standing) produce an acceleration vector magnitude approximately $1.0 \mathrm{~g}$ while they produce above $1.5 \mathrm{~g}$ in active state. In this study we used $1.6 \mathrm{~g}$ as threshold and the active state was calculated using a non-overlapped 2 seconds sliding window [16].

\subsubsection{Minimum Acceleration (Min)}

The minimum vector magnitude of acceleration over $1 \mathrm{sec}-$ ond window was calculated. Based on Kangas et al. [1], the minimum value of vector magnitude was nearly $0.0 \mathrm{~g}$ in a pre-impact fall event.

\subsubsection{Maximum Acceleration (Max)}

The maximum vector magnitude of acceleration over 1 second overlapping window was calculated. It overlapped by $50 \%$ from the Minimum Value feature window. This feature was used to detect the highest peak of the vector magnitude of the acceleration when the fall impact phase happened [1].

\subsubsection{Mean}

Based on Ojetola [16], the mean acceleration is higher in preimpact and impact stages than the post impact. Therefore this feature is calculated for those three stages.

\subsubsection{Velocity}

We used this feature because the velocity value from fall events tends to be higher than static activities [4]. This feature was calculated for the three stages. The equation to calculate this feature is:

$$
V=\int V_{m} d t
$$



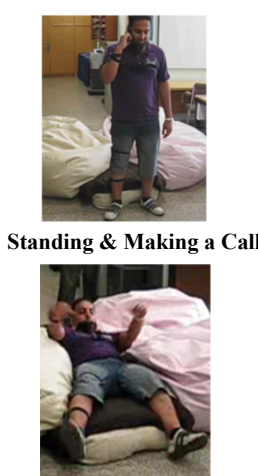

Falling Backward

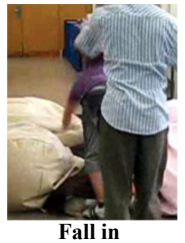

Left Direction
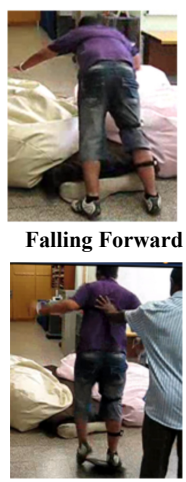

Near Fall

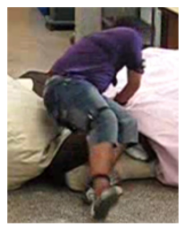

Pushed to Fall Forward

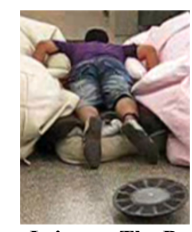

Lying on The Bed

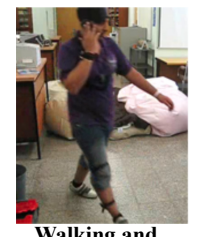

Walking and

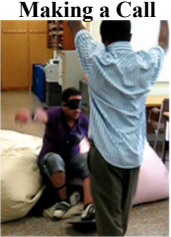

Pushed to Fall Backward

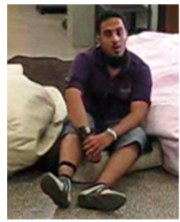

Sitting on The Bed

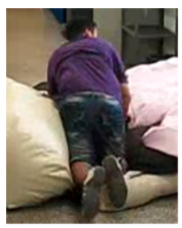

Crouching

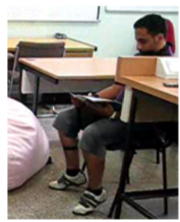

Sitting on The Chair

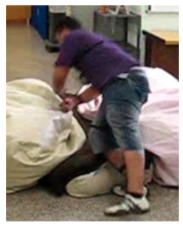

Fall in

Right Direction

Figure 1: Activities of Daily Living (ADLs) and simulated fall illustration[16]

where $V_{m}$ is the acceleration vector magnitude.

\subsubsection{Energy Expenditure (Energy)}

The energy expenditure was calculated for the three stages [16]. The energy expenditure was calculated using:

$$
E=\int a_{x}^{2} d t+\int a_{y}^{2} d t+\int a_{z}^{2} d t
$$

where $a_{x}, a_{y}$, and $a_{z}$ are the outputs of the accelerometer on x-axis, y-axis, and z-axis respectively.

\subsubsection{Variance}

The variance of accelerometer vector magnitude was calculated for the three stages $[5,16]$.

\subsubsection{Root Mean Square (RMS)}

The RMS of the acceleration signal was also calculated for the pre-impact, impact, and post-impact stages. RMS has previously been used in research for fall detection $[4,9]$.

\subsubsection{Exponential Moving Average (EMA)}

The EMA value of vector magnitude was calculated from the three stages as feature values [5]. EMA is calculated as:

$$
s_{t}=\alpha V_{m}+(1-\alpha) s_{t-1},
$$

where $s_{t}$ is the EMA value at time $t$, is the smoothing factor, and $V_{m}$ is the vector magnitude of acceleration.

\subsubsection{Signal Magnitude Area (SMA)}

The SMA is useful to distinguish human activities [12]. It was calculated using:

$$
\gamma=\frac{1}{t}\left(\int_{0}^{t}\left|a_{x}\right| d t+\int_{0}^{t}\left|a_{y}\right| d t+\int_{0}^{t}\left|a_{z}\right| d t\right),
$$

Table 2: Fall stages and their extracted features

\begin{tabular}{ccc}
\hline Pre-impact & Impact & Post-impact \\
\hline Mean & Mean & Mean \\
Velocity & Velocity & Velocity \\
Energy Expenditure & Energy Expenditure & Energy Expenditure \\
Variance & Variance & Variance \\
Root Mean Square & Root Mean Square & Root Mean Square \\
Exponential Moving Average & Exponential Moving Average & Exponential Moving Average \\
Signal Magnitude Area & Signal Magnitude Area & Signal Magnitude Area \\
Minimum Acceleration & Maximum Acceleration & \\
\hline
\end{tabular}

where $\gamma$ is the SMA value and $a$ is the accelerometer value from $x, y$, and $z$ axis.

The stages and their respective features are shown in Table 2 .

\subsection{The Cascade-Classifier Approach}

The key aspect of the cascade-classifier approach is the use of a threshold to trigger the feature calculation process. In this way, the cascade-classifier approach prevents the system performing the features calculation for all samples. Our proposed cascade-classifier approach is shown in Algorithm 1.

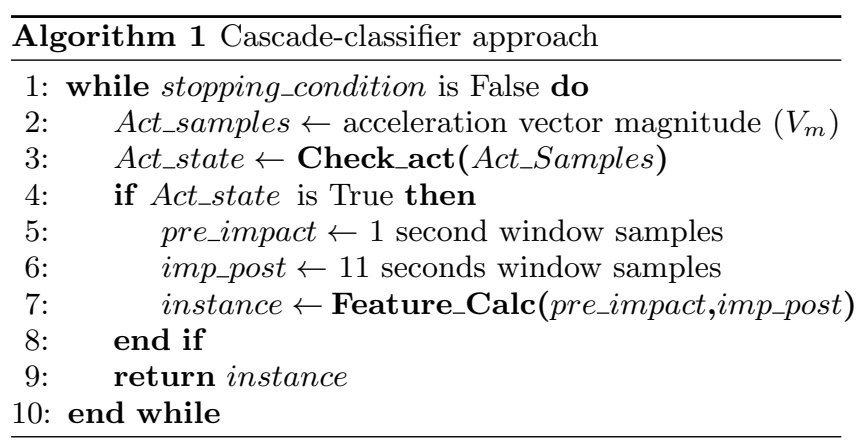




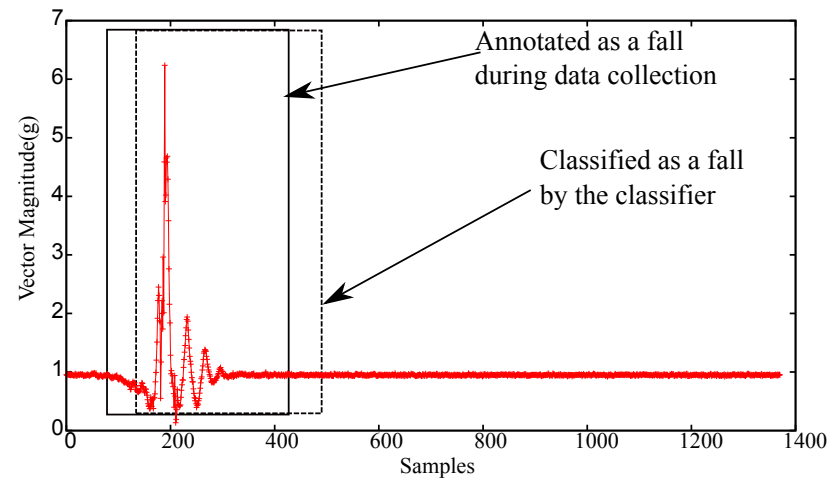

(a) Ambiguity in window-based annotation

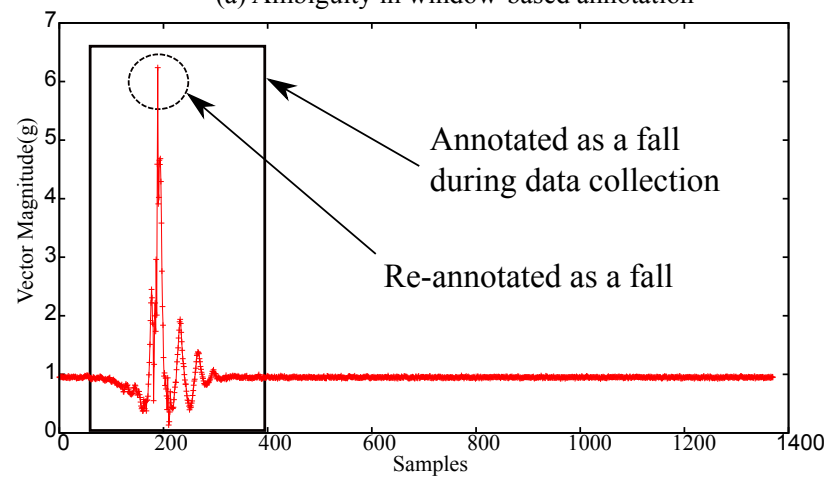

(b) Micro-annotation approach

Figure 2: (a) Ambiguity in sliding window based approach; (b) Micro-annotation approach

Active State (Check_act()) of the samples using a nonoverlapped sliding window every 2 seconds. This means, if a sample that has a vector magnitude of acceleration higher than $1.6 \mathrm{~g}$ is detected, the features calculation process is triggered. We call this sample the active sample. Otherwise, the feature calculation process is not triggered.

The second phase contains features calculations. This process is started by collecting samples with a 1 second window before and 11 seconds window after the active sample. The 1 second window before the active sample captures the preimpact stage while the 11 seconds window after the active sample captures the impact and post-impact stages. The next step calculates the features based on the three stages (pre-impact, impact, and post-impact). The features and their windows are illustrated in Figure 3.

\section{EXPERIMENTAL EVALUATION \\ 5.1 Experimental Setup}

The data were pre-processed offline using Python programming language and WEKA [10] was chosen for training and testing the instances. J48 Decision Tree, Logistic Regression and Multilayer Perceptron were used as the classifier. For the Multilayer Perceptron classifier, we used 3 hidden layers. Five nodes were used in the first hidden layer, four nodes in the second hidden layer, and three nodes were used in the third hidden layer. As comparison, we also implemented a sliding window based approach that uses J48 Decision Tree as a classifier [16]. We used a machine with the specification: Intel core i7 @ $3.10 \mathrm{GHz}, 8 \mathrm{~GB}$ of DDR3 Memory, and

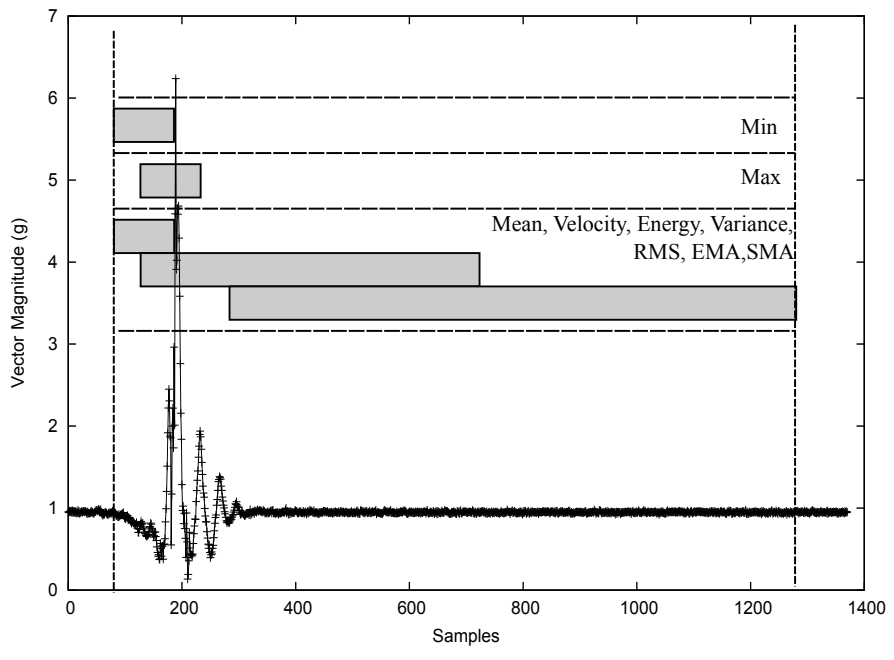

Figure 3: Features window illustration

Table 3: Precision, Recall, and f-measure from sliding window based J48 Decision Tree (J48 DT), cascade-classifier based J48 Decision Tree (CC J48 DT ), cascade-classifier based Logistic Regression (CC LR), and cascade-classifier based Multilayer Perceptron (CC MLP)

\begin{tabular}{lllllll}
\hline \multirow{2}{*}{ Classifier } & \multicolumn{2}{l}{ Precision (\%) } & \multicolumn{2}{l}{ Recall (\%) } & \multicolumn{2}{c}{ f-measure (\%) } \\
\cline { 2 - 7 } & Mean & STD & Mean & STD & Mean & STD \\
\hline J48 DT & 86.9 & 9.5 & 89.0 & 13.6 & 87.4 & 10.1 \\
CC J48 DT & 87.5 & 10.8 & 86.0 & 17.7 & 85.7 & 13.6 \\
CC LR & 90.8 & 6.7 & 90.8 & 13.4 & 90.0 & 8.5 \\
CC MLP & 93.5 & 7.0 & 94.2 & 8.92 & 93.5 & 6.3 \\
\hline
\end{tabular}

Linux Mint 17 Qiana to run the experiment.

Leave-one-subject-out cross-validation was used in this experiment. Precision, recall, and f-measure were used to measure the performance of our cascade-classifier. The exact Wilcoxon sign rank test was performed to show the significance of our improvement.

\subsection{Results and Discussion}

The result of our experiment shows that our cascade-classifier can achieve up to $93.5 \%$ of f-measure when it used Multilayer Perceptron as the classifier. Our approach can surpass the sliding window based approach's accuracy significantly if Logistic Regression $(p$-value $=0.04)$ or Multilayer Perceptron $(p$-value $=0.00001)$ are used as the classifier. Although our cascade-classifier approach with J48 Decision Tree as a classifier achieves lower performance than the sliding window based algorithm, the difference is not significant $(p$-value $=0.3)$. Overall, the Multilayer Perceptron is the best classifier for our cascade-classifier approach because it achieves the highest f-measure. Table 3 shows the average mean and standard deviation of the precision, recall, and f-measure of the sliding window based algorithm and our cascade-classifier approach. The distribution of the performance values from all classifiers is shown in Figure 4.

In our experiment, the running time of the feature calculation processes were computed to show that our cascadeclassifier approach is able to reduce the computational cost 


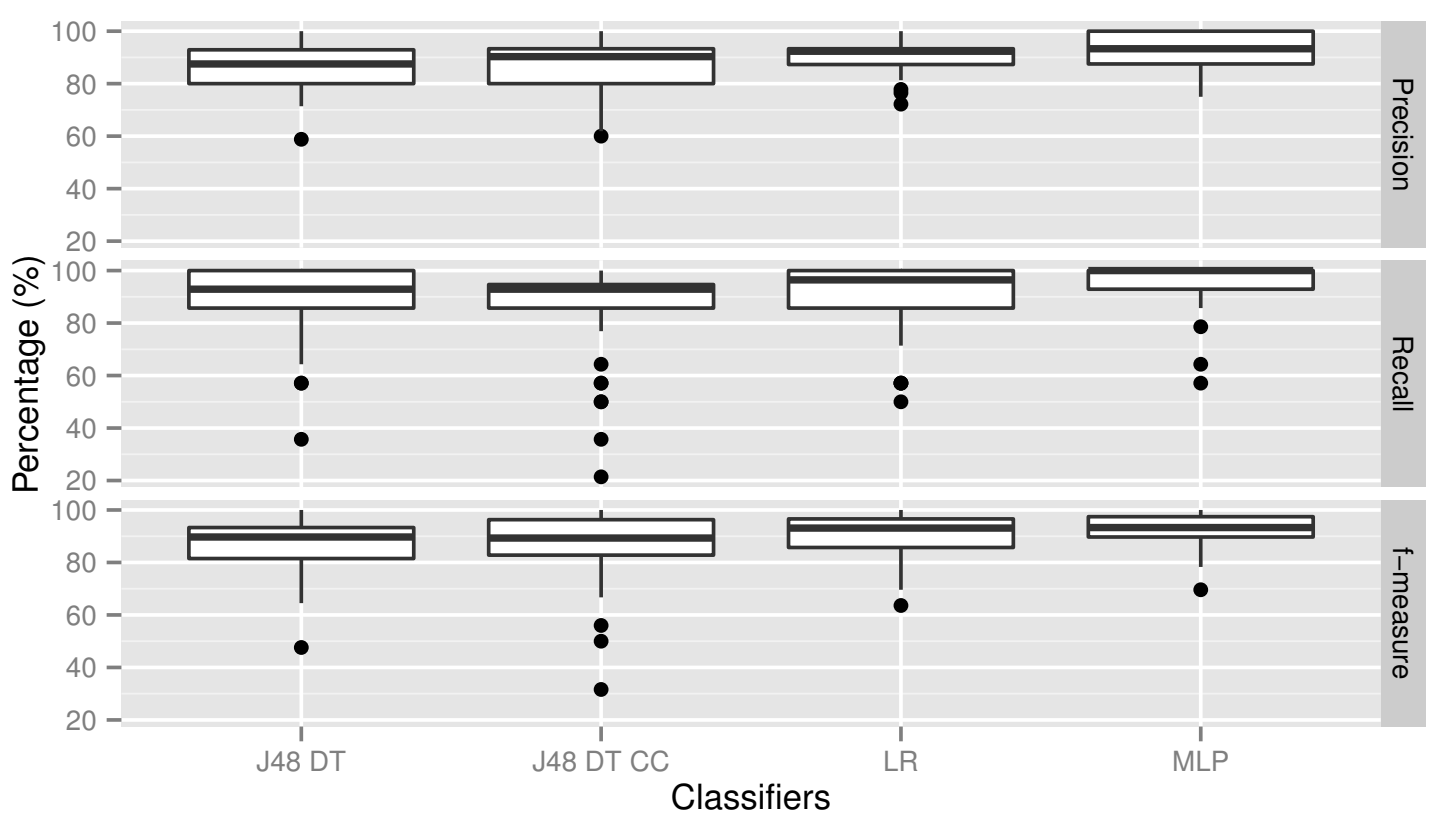

Figure 4: The classifiers performance

Table 4: Running time

\begin{tabular}{ccccc}
\hline \multirow{2}{*}{ Approach } & \multicolumn{2}{c}{$\begin{array}{c}\text { A segment } \\
\text { from a subject }\end{array}$} & \multicolumn{2}{c}{$\begin{array}{c}\text { All segments } \\
\text { from a subject }\end{array}$} \\
\cline { 2 - 5 } & Average & STD & Average & STD \\
\hline Sliding window & $0.025 \mathrm{~s}$ & 0.0009 & $2677.1 \mathrm{~s}$ & 712.7 \\
Cascade-classifier & $0.006 \mathrm{~s}$ & 0.0006 & $0.4 \mathrm{~s}$ & 0.05 \\
\hline
\end{tabular}

of the machine learning approach. As all the subjects in the dataset follow the same protocol, a subset of them that consists of 4 random subjects was used. The experiment measured the time that was needed to calculate features for each segment and all segments from a subject.

Table 4 shows that our cascade-classifier approach requires significantly less time than the sliding window based approach for feature extractions of each segment $(p$-value $=$ $3.327 \mathrm{e}-09)$ and all segments $(p$-value $=3.327 \mathrm{e}-09)$. The delay in detecting falls can be reduced once the running time of the feature calculation from a segment is reduced. By detecting the falls early, the negative effects of them can be reduced. Furthermore, our approach also requires less time to do feature calculation from all segments corresponding to one subject than the sliding window based approach. This means that if our algorithm is implemented on the node, it prevents the sensor node battery lifetime from draining rapidly.

\section{CONCLUSION AND FUTURE WORK}

This study proposed a cascade-classifier approach to overcome the high computational cost issue in machine learning based algorithms for fall detection. Our cascade-classifier approach uses ACTIVE STATE feature in the initial phase to trigger the features calculation in the next phase. This technique prevents the features calculation being executed for some samples. As classifiers, this study used J48 Decision Tree, Logistic Regression, and Multilayer Perceptron. A large dataset of Falls and ADLs with total of 48 subjects and 672 simulated falls was used to assess our approach. We also implemented a sliding window based machine learning approach for a comparison.

From our experiment, it can be concluded that the cascadeclassifier approach can significantly reduce the computational time of the current sliding window based machine learning algorithm. It can lead to reduction in computational cost and have a further impact on energy consumption of the node. In terms of accuracy, our approach is able to achieve better performance than the sliding window based machine learning algorithm if it uses Logistic Regression or Multilayer Perceptron as the classifier. Among the classifiers, the Multilayer Perceptron achieves the best performance with $93.5 \%$ of precision, $94.5 \%$ of recall, and 93.5 of f-measure.

Some possible avenues for the next step of our research are: (i) investigation of postural sway [19] to trigger the feature calculation process; (ii) investigation of low-cost features that can increase both performance and speed of the classifier.

\section{Acknowledgment}

The authors would like to thank to Dr Olukunle Ojetola for his valuable input.

\section{REFERENCES}

[1] S. Abbate, M. Avvenuti, F. Bonatesta, G. Cola, P. Corsini, and A. Vecchio. A smartphone-based fall detection system. Pervasive and Mobile Computing, 8(6):883-899, 2012. 
[2] Age UK. Stop falling: Start saving lives and money. http:

//www. ageuk.org.uk/documents/en-gb/campaigns/ stop_falling_report_web.pdf?dtrk=true, 2013.

[3] F. Bagalà, C. Becker, A. Cappello, L. Chiari, K. Aminian, J. M. Hausdorff, W. Zijlstra, and J. Klenk. Evaluation of accelerometer-based fall detection algorithms on real-world falls. PLoS ONE, $7(5): 1-9,2012$.

[4] A. K. Bourke, K. J. O'Donovan, J. Nelson, and G. M. OLaighin. Fall-detection through vertical velocity thresholding using a tri-axial accelerometer characterized using an optical motion-capture system. Conference proceedings : ... Annual International Conference of the IEEE Engineering in Medicine and Biology Society. IEEE Engineering in Medicine and Biology Society. Conference, 2008:2832-2835, 2008.

[5] J. Brusey, R. Rednic, E. I. Gaura, J. Kemp, and N. Poole. Postural activity monitoring for increasing safety in bomb disposal missions. Measurement Science and Technology, 20(7):075204, 2009.

[6] Y. Choi, a. S. Ralhan, and S. Ko. A study on machine learning algorithms for fall detection and movement classification. In 2011 International Conference on Information Science and Applications, ICISA 2011, pages 1-8, Jeju Island, Republic of Korea, 2011. IEEE.

[7] C. Dinh and M. Struck. A new real-time fall detection approach using fuzzy logic and a neural network. In Wearable Micro and Nano Technologies for Personalized Health (pHealth), 2009 6th International Workshop on, pages 57-60, June 2009.

[8] J. H. Downton. Falls in the Elderly. Edward Arnold London, 1993.

[9] H. Gjoreski, M. Lustrek, and M. Gams. Accelerometer Placement for Posture Recognition and Fall Detection. In 2011 Seventh International Conference on Intelligent Environments, pages 47-54. Ieee, July 2011.

[10] M. Hall, E. Frank, G. Holmes, B. Pfahringer, P. Reutemann, and I. H. Witten. The weka data mining software: An update. SIGKDD Explor. Newsl., 11(1):10-18, Nov. 2009.

[11] R. Igual, C. Medrano, and I. Plaza. Challenges, issues and trends in fall detection systems. Biomedical engineering online, 12:66, 2013.

[12] D. M. Karantonis, M. R. Narayanan, M. Mathie, N. H. Lovell, and B. G. Celler. Implementation of a real-time human movement classifier using a triaxial accelerometer for ambulatory monitoring. IEEE Transactions on Information Technology in Biomedicine, 10(1):156-167, 2006.

[13] L. J. Kau and C. S. Chen. A smart phone-based pocket fall accident detection system. 2014 IEEE International Symposium on Bioelectronics and Bioinformatics, IEEE ISBB 2014, 19(1):44-56, 2014.

[14] J. K. Lee, S. Robinovitch, and E. Park. Inertial Sensing-Based Pre-Impact Detection of Falls Involving Near-Fall Scenarios. IEEE Transactions on Neural Systems and Rehabilitation Engineering, 4320(c):1-1, 2014.

[15] A. J. A. Majumder, I. Zerin, S. I. Ahamed, and R. O. Smith. A multi-sensor approach for fall risk prediction and prevention in elderly. ACM SIGAPP Applied
Computing Review, 14(1):41-52, 2014.

[16] O. Ojetola. Detection of Human Falls using Wearable Sensors. PhD thesis, 2013.

[17] O. Ojetola, E. Gaura, and J. Brusey. Data set for fall events and daily activities from inertial sensors. In Proceedings of the 6th ACM Multimedia Systems Conference, MMSys '15, pages 243-248, New York, NY, USA, 2015. ACM.

[18] O. Ojetola, E. I. Gaura, and J. Brusey. Fall detection with wearable sensors - Safe (SmArt Fall dEtection). In Proceedings - 2011 7th International Conference on Intelligent Environments, IE 2011, pages 318-321, 2011.

[19] A. Turcato and S. Ramat. Predicting losses of balance during upright stance: Evaluation of a novel approach based on wearable accelerometers. In 2010 Annual International Conference of the IEEE Engineering in Medicine and Biology Society, EMBC'10, pages 4918-4921, 2010

[20] M. Vallejo, C. V. Isaza, and J. D. Lopez. Artificial Neural Networks as an alternative to traditional fall detection methods. In Conference proceedings : ... Annual International Conference of the IEEE Engineering in Medicine and Biology Society. IEEE Engineering in Medicine and Biology Society. Conference, volume 2013, pages 1648-51, 2013.

[21] G. Wu and S. Xue. Portable preimpact fall detector with inertial sensors. IEEE Transactions on Neural Systems and Rehabilitation Engineering, 16(2):178-183, 2008. 